\title{
Information Entropy of Single-Gene Expression Responses during Genome Wide Perturbations ${ }^{\dagger}$
}

\author{
Bilena Almeida, Mohamed Bahrudeen, Vatsala Chauhan, Cristina Palma, Ines Baptista, Suchintak
} Dash, Vinodh Kandavalli and Andre Ribeiro

Tampere University, Tampere, Finland

† Presented at the Entropy 2021: The Scientific Tool of the 21st Century, 5-7 May 2021; Available online: https://sciforum.net/conference/Entropy2021/.

Published: 5 May 2021

Transcription factors (TF) are known to drive gene-to-gene interaction dynamics under optimal growth conditions, but lesser is known about how much they affect the dynamics of gene regulatory networks (GRN) at the global level, due to the contribution of many other variables.

We investigate how TF interactions of the GRN of E. coli affects the global entropy of single-genes response dynamics, during a genome-wide perturbation caused by a shift in RNA polymerase (RNAp) concentrations.

For this, we classified genes based on their number of (known) input TFs. Also, we assigned a value to each TF input ( -1 for repression and +1 for activation) and classified genes based on the sum of its input interactions. For both classification schemes, we estimated the information entropy of the single-gene input interactions of each class.

Next, we measured by RNA-seq the fold changes of each gene due to weak, medium, and strong pervturbations of RNAp concentration, from which we quantified the information entropy of singlegene responses of each class.

We found that the information entropy of the fold changes of the classes of genes increases (nonlineVarly) with the magnitude of the perturbation, in a manner that is consistent with the information entropy of the sum of the input interactions of individual genes, rather than their number of inputs.

Overall, we argue that, in the event of genome wide perturbations, asymmetries in input functions of TFs partially control the propagation of information between genes of the GRN of E. coli.

(C) 2021 by the authors. Licensee MDPI, Basel, Switzerland. This article is an open access article distributed under the terms and conditions of the Creative Commons Attribution (CC BY) license (http://creativecommons.org/licenses/by/4.0/). 